\title{
Optimization analysis of pre tightening pressure of NC rebar length measure system based on response surface methodology
}

\author{
Shenghao Tong ${ }^{a}$, Ke Zhang ${ }^{b}$, Songhua $\mathrm{Li}^{\mathrm{c}}$, Yanze Long ${ }^{\mathrm{d}}$ \\ Shenyang Jianzhu University, Shenyang 110168, China \\ awtongshenghao@163.com, brzhangke@sjzu.edu.cn, crick_li2000@qq.com, d692476254 @qq.com
}

Keywords: Rebar straightening machine, length measure, pre tightening pressure.

\begin{abstract}
The pre tightening pressure of the length measuring wheel was optimized by response surface methodology in the self-developed NC length measure of bar system, the pre tightening pressure and external load are set as the input parameter variable, the stress of the length measuring wheel is set as an output parameter variable, establish continuous variable surface model. Evaluate the interactive factors that influence the vibration and wear of the length measuring wheel, make up for the deficiency of the traditional single variable optimization analysis. Using the response surface method analysis the influence of pre tightening pressure and external load force on the length measuring wheel, the result shows that the pre tightening force has great influence on the stress of the length measuring wheel, while the external load force has little influence on it, so it is showed that there is a great relationship between the wear and pre tightening pressure of the length measuring wheel, get the optimal preload value, the reference is provided for the operator of the straightening machine.
\end{abstract}

\section{Introduction}

In recent years, with the rapid development of the national economy, the state has increased investment in infrastructure construction. As an efficient steel processing equipment is widely used in the construction field, it straightening and cut a plate of rebar into sections in accordance with the length need, length measure of rebar is completed by the length measure system, so the measuring precision and reliability of reinforced determine the machine performance. The traditional scaling mechanism adopts touch switch, this method not only makes the switch contact wear, and it can't change the cutting length, brought great distress to the processing workers. Independently developed NC rebar measure system, the use of three pairs of rollers, vertical rail adjustable, belt wheel shock absorber, encoder count and other functions to achieve the measuring of the high-precision steel rebar. Response surface regression analysis is a method of optimization process, using this method can not only establish a continuous variable surface model, but also the formation of response surface, to evaluate the factors influencing the process and their interaction, to make up for the traditional single factor experiment is not enough.

\section{Model Establishment and Analysis}

Length measure system structure as shown in Figure 1, Straightening rebar into the measure system, measure wheel press on the rebar under the action of pre tightening pressure, reinforced in forward motion at the same time, foot wheel rotation and transmit the signal to the rotary encoder through the damping pulley group encoder.

With the diameter of $10 \mathrm{~mm}$ steel as an example, in order to increase the friction, a lot of convex processing round the outer edge of measuring wheel contact with reinforced with outer rib, the angle between the position which measure wheel contact rebar and the vertical centerline of measure wheel is $\theta$. The size and the distance of the outer convex platform of the measure wheel determine the direction of the external load, and the pressure direction of the pre tightening is vertically downward. The relationship between preload and external load is calculated by using Descartes coordinate system and its transformation coordinate system. 


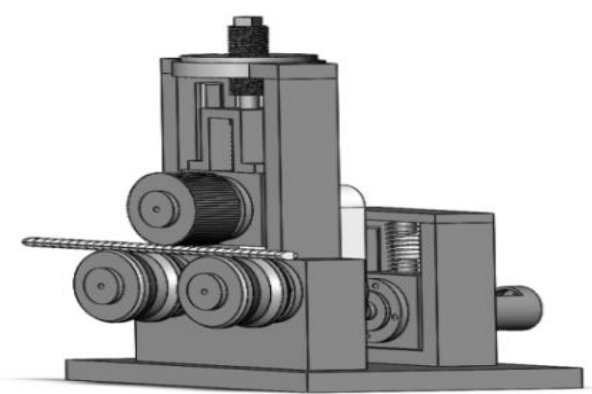

Fig. 1 Numerical control length measuring system

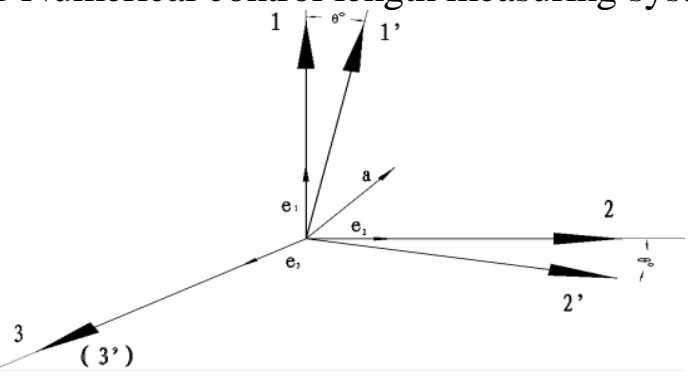

Fig. 2 Descartes coordinate system and its conversion coordinate system

The Cartesian coordinate system and its transformation coordinate system are shown in figure 3 , the 1'2'3' coordinate system is the pre - tightening coordinate system, which is denoted as the $\mathrm{X}_{\mathrm{i}}$ coordinate system, the 1'2'3'coordinate system is the external load coordinate system, which is denoted as the $\mathrm{X}_{\mathrm{i}}$ coordinate system, and the angle between the axis of the 1 and the 1 axis of the two coordinate system between the 2 coordinate system and the 2 axis is. Two coordinate systems $\mathrm{X}_{\mathrm{i}}$ and $\mathrm{X}_{\mathrm{i}}$ 'orthonormal basis $e_{i}$ and $e_{i}^{\prime}$, the new basis $e_{i}^{\prime}$ on the old basis $e_{i}$ decomposition.

$$
e_{i}^{t}=\beta_{i 11} e_{1}+\beta_{i r 2} e_{2}+\beta_{i r a} e_{a}=\beta_{i j i} e_{i}
$$

Among them, e1, e2, e3 are the basis vectors in the Xi coordinate system.

$\beta_{\mathrm{i} j \mathrm{j}}=\cos \left(\mathrm{e}_{\mathrm{i}}^{\mathrm{t}}, \mathrm{e}_{\mathrm{j}}\right)=\mathrm{e}_{\mathrm{i}}^{t} \times \mathrm{e}_{\mathrm{j}}=\mathrm{e}_{\mathrm{j}} \times \mathrm{e}_{\mathrm{i}}^{t}$

It is the cosine of the angle between the new and old $\mathrm{x} \_\mathrm{i}^{\wedge}$ 'axis $\mathrm{x} \_\mathrm{i}$ axis, known as conversion factor.

The new expression of $X_{i}$ 'is represented by the old coordinate for $X_{i}$ $\mathrm{x}_{\mathrm{i}}^{f}=\beta_{\mathrm{i} i \mathrm{j}} \mathrm{x}_{\mathrm{j}}+\left(\mathrm{x}_{\mathrm{i}}^{\mathrm{f}}\right)_{0}$

Among them, "_0" $\left(\mathrm{x}_{-} \mathrm{i}^{\prime}\right)$ is a new and old origin $\mathrm{O}$ value. The matrix form is

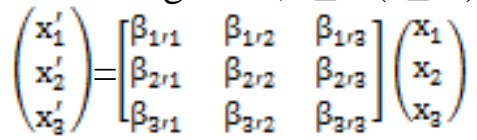

$$
\begin{aligned}
& \beta_{1 / 1}=\cos \theta, \beta_{1 / 2}=\cos \theta, \beta_{1 / a}=0, \beta_{2 r 1}=-\sin \theta, \beta_{2 r 2}=\cos \theta, \beta_{2 / a}=0, \beta_{211}=0, \beta_{2 r 2}=0, \beta_{2 r a}=1 \text {. }
\end{aligned}
$$

\section{Optimization Analysis}

\subsection{Parameterized Variable Settings}

The parametric CAD model is loaded and solved. The pre tightening force and the external load direction are taken as input parameters, and the stress and strain are taken as the output parameters. The component of the external load in the vertical direction is the same, so the direction of the resultant force is determined by the size of the component in the parallel direction. The transmission parameter variable is set as shown in Figure 4, P3, P4 is the input parameter variable, P1, P2 is the output parameter variable. 


\begin{tabular}{|c|c|c|}
\hline & A & B \\
\hline 1 & & Enabled \\
\hline 2 & $\square \checkmark$ Design of Experiments & \\
\hline 3 & $\square$ Input Parameters & \\
\hline 4 & $\square$ Static Structural (A 1) & \\
\hline 5 & [P P3 - Force Y Component & 四 \\
\hline 6 & [p P4 - Force 2 Y Component & $\square$ \\
\hline 7 & $\square \quad$ Output Parameters & \\
\hline 8 & $\square \square$ Static Structural (A 1) & \\
\hline 9 & Pat P1 - Equivalent Stress Maximum & \\
\hline 10 & P. P2 - Total Deformation Maximum & \\
\hline 11 & P. P5 - Geometry Mass & \\
\hline 12 & $\square$ Charts & \\
\hline 13 & $\checkmark N$ Parameters Parallel & \\
\hline 14 & $\checkmark W$ Design Points vs Parameter & \\
\hline
\end{tabular}

\subsection{Results After Treatment}

Fig.3 Input parameter variable and output parameter variable

The initial value and the upper and lower limits of the parameterized variable are given according to the experience, and the results of the 9 design points are listed in the DOE table after calculation, as shown in figure 5. DOE list second or third column value of the input parameter range of values for the experience of fourth columns and fifth columns for output parameter variables, the first row in a data table for the initial value, can be seen examining round before optimization stress is $1.1841 \times 10^{8} \mathrm{~Pa}$. Table of Schematic B2: Design of Experiments (Central Composite Design : Auto Defined)

\begin{tabular}{|c|c|c|c|c|c|}
\hline & A & B & c & D & E \\
\hline 1 & 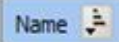 & P3 - Force Y Component $(\mathbb{N}) \quad \checkmark$ & P4 - Force $2 Y$ Component $(N)$ V & $P_{1}$ - Equivalent Stress Maximum $(\mathrm{Pa})$ & P2 - Total Deformation Maximum $(m)$ \\
\hline 2 & 1 & -1181.7 & 1181.7 & $1.1841 E+08$ & $3.551 E-05$ \\
\hline 3 & 2 & -1299.9 & 1181.7 & $1.1819 E+08$ & 9.9316 \\
\hline 4 & 3 & -1063.5 & 1181.7 & $1.1863 E+08$ & 9.9316 \\
\hline 5 & 4 & -1181.7 & 1063.5 & $9.5122 E+07$ & 9.9316 \\
\hline 6 & 5 & -1181.7 & 1299.9 & $1.417 E+08$ & 9.9317 \\
\hline 7 & 6 & -1299.9 & 1063.5 & $9.7425 E+07$ & 19.863 \\
\hline 8 & 7 & -1063.5 & 1063.5 & $9.5344 E+07$ & $2.8487 E-05$ \\
\hline 9 & 8 & -1299.9 & 1299.9 & $1.414 \pi+08$ & $4.2534 E-05$ \\
\hline 10 & 9 & -1063.5 & 1299.9 & $1.4192 \mathrm{E}+08$ & 19.863 \\
\hline
\end{tabular}

Fig.4 List of calculated results for design points

In order to directly see the DOE table nine data points can be compared, the stress of each design point and its corresponding value curve diagram, as shown in figure 6 . It can be seen from the figure 4 design points corresponding to the lowest stress value, from the design point of the calculation results found design list 4 component loads corresponding to the $1181.7 \mathrm{~N}$, the pretightening force is $1063.5 \mathrm{~N}$, the stress value is $9.5122 \times 10^{7} \mathrm{~Pa}$, less than $1.1841 \times 10^{8}$.

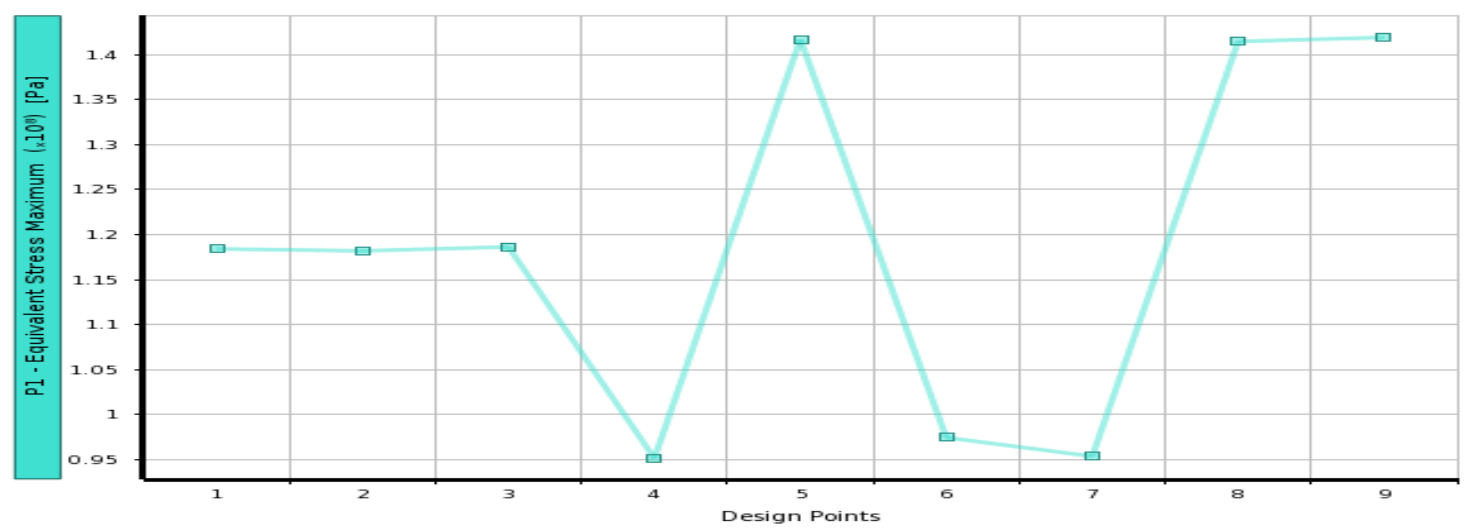

Fig.5 Design point stress value curve

\subsection{Parameter Variable Evaluation}

The stress value is $\mathrm{Z}$ axis coordinate, the pre tightening force value is $\mathrm{X}$ axis coordinate, the external load component is $\mathrm{Y}$ axis coordinate to establish the response surface, as shown in figure 7 . It can be 
seen from the figure measuring wheel by changes in the $\mathrm{X}$ direction stress is large, and in the $\mathrm{Y}$ direction of small changes, that foot wheel preload on the effect of stress is large, but the direction of the stress load has little influence on the foot wheel. In this paper, the pre stress value corresponding to the minimum value of the $\mathrm{Z}$ axis of the response surface is the best pre tightening force that we want to obtain, and the optimal preload value is $1063.5 \mathrm{~N}$.

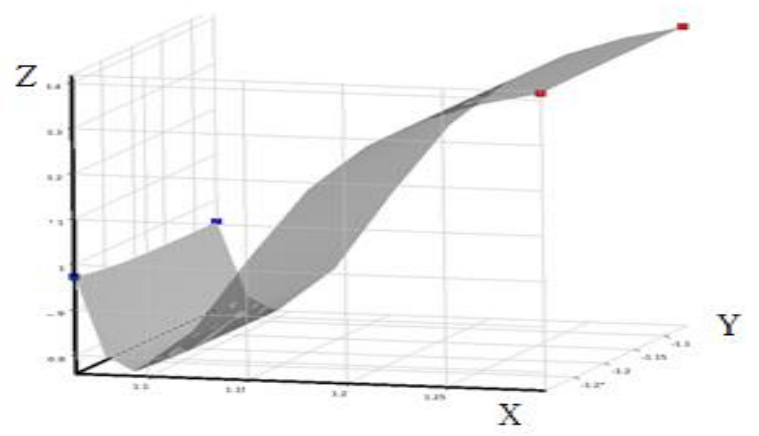

Fig.6 The influence of pre tightening pressure and external load force on the length measuring wheel

\section{Summary}

Establish contact model of measure wheel and rebar, use the Descartes coordinate and coordinate transformation to obtain the relationship between preload and external load, reflects the contact condition between measure wheel and rebar, lay the foundation for optimization analysis. The preload of measure wheel and external load force as input parameters, the stress as output parameters, make optimization analysis, obtain optimum preload value, and provide a reference for the straightening machine operator. Use the response surface method to analysis influence of preload and external load on the measure wheel, which show that the preload has great influence on the stress of measure wheel, while the load force has little effect on it, it indicates that there is a lot of relationship between the wear of measure wheel and pre tightening force.

\section{Acknowledgments}

National Joint Engineering Laboratory Project, SJSC-2015-17.

\section{References}

[1]. DeMeo M, Laget M, Mathieu D. Application of experimental designs for optimization of medium and culture conditions in fermentation[J]. Bioscience, 1985, 4: 99-102.

[2]. WANG H T, LIU Z J, CHEN S X. Application of Taguchi method to robust design of BLDC motor performance [J]. IEEE Transactions on Magnetics, 1999, 35(5):3700 - 3702.

[3]. RAJEMI M F, MATIVENGA P T, ARAMCHAROEN A sustainable machining: Selection of optimum turning conditions based on minimum energy considerations [J]. Journal of Cleaner Production 2010(18):1059-1065.

[4]. WANG J, KAMPE M J, Van der Westhuizen K, et al. Optimal design of a coreless stator axial flux permanent magnet generator [J]. IEEE Transactions on Magnetics, 2005, 41(1): 55 - 64 .

[5]. Harris C, Despa M, Kelly K. Design and fabrication of a cross flow micro heat exchanger [J]. Journal of Micro electro mechanical Systems, 2000, 9(4):502-508. 